\title{
Immune checkpoint inhibitors in gastrointestinal malignancies: what can we learn from experience with other tumors?
}

\author{
Anand B. Shah ${ }^{1}$, Katelyn R. Sommerer ${ }^{1}$, Khaldoun Almhanna ${ }^{2}$ \\ ${ }^{1}$ Department of Pharmacy, Moffitt Cancer Center, Tampa, FL, USA; ${ }^{2}$ Division of Hematology/Oncology, The Warren Alpert Medical School of \\ Brown University, Lifespan Cancer Institute, Rhode Island Hospital, Providence, RI, USA \\ Contributions: (I) Conception and design: AB Shah, K Almhanna; (II) Administrative support: None; (III) Provision of study materials or patients: \\ None; (IV) Collection and assembly of data: None; (V) Data analysis and interpretation: None; (VI) Manuscript writing: All authors; (VII) Final \\ approval of manuscript: All authors. \\ Correspondence to: Anand B. Shah, PhD, BCOP, BCPS. Department of Pharmacy, Moffitt Cancer Center, 12902 Magnolia Drive, Tampa, FL 33612, \\ USA. Email: anand.shah@moffitt.org.
}

\begin{abstract}
Gastrointestinal (GI) malignancies are some of the most common cancers worldwide with high rates of morbidity and mortality. Immune checkpoint inhibitors have afforded additional treatment options for patients, but their success has been limited. Conversely, in other tumor types such as lung cancer, melanoma and renal cell carcinoma, treatment strategies with immune checkpoint inhibitors have propelled those agents into the front lines of treatment. Strategies utilized include combining immune checkpoint inhibitors with chemotherapy, other checkpoint inhibitors, and targeted therapy. In this review, we analyze combination strategies employed in other tumor types to help identify current and future approaches toward improving outcomes with immunotherapy in GI malignancies.
\end{abstract}

Keywords: Immunotherapy; checkpoint inhibitor; gastrointestinal cancer (GI cancer); combination

Received: 21 August 2019; Accepted: 12 September 2019; Published: 10 October 2019.

doi: $10.21037 /$ tgh.2019.09.04

View this article at: http://dx.doi.org/10.21037/tgh.2019.09.04

\section{Introduction}

Gastrointestinal (GI) malignancies include cancers of the esophagus, gallbladder, liver, pancreas, stomach, small intestine, large intestine and the anus. Together, GI malignancies comprise some of the most prevalent cancer types worldwide and also account for a large number of global deaths (1). Treatment of GI tumors consists of a multidisciplinary approach, including surgery, radiation and chemotherapy $(2,3)$. These approaches have improved outcomes in some patients, but the management of patients with metastatic disease remains challenging. New strategies are desperately needed.

It has been long suspected that the immune system plays an important role in the development and progression of cancers (4). Suppression of the tumor microenvironment (TME) and evasion from the immunosurveillance system allow malignant cells to develop into tumors (5). Current research is targeted at reversing immune suppression in the TME as well as uncloaking cancer cells to enable the immune system to recognize them as foreign and destroy them. Immune checkpoints are cell surface receptors expressed by immune cells that regulate the activation of $\mathrm{T}$ lymphocytes (6). These co-stimulatory and co-inhibitory checkpoint molecules act as gatekeepers of the immune response. Checkpoints that have been a major focus in research include the cytotoxic $\mathrm{T}$ lymphocyte antigen-4 (CTLA-4) interaction with B7 and the programmed death-1 $(\mathrm{PD}-1)$ interaction with programmed death-ligand 1 (PD-L1).

In 2011, the first checkpoint inhibitor approved by the FDA was ipilimumab, a CTLA-4 antibody (7). Since then, immunotherapies have been successfully used to treat various solid tumor malignancies. For example, long lasting responses are now possible for patients with metastatic melanoma (8). Similar successes have been noted in renal 
cell carcinoma and lung cancer $(9,10)$. Several immunecheckpoint inhibitors have been approved by the FDA. These include ipilimumab (anti-CTLA4); nivolumab, pembrolizumab and cemiplimab (anti-PD-1); and avelumab, durvalumab and atezolizumab (anti-PD-L1).

Immunotherapy, especially checkpoint inhibitors, has been used in GI malignancies. Currently, FDA-approved indications include: nivolumab and pembrolizumab for second-line treatment of advanced hepatocellular carcinoma (HCC) (11,12); nivolumab and pembrolizumab for microsatellite instability-high (MSI-H) or mismatch repair deficient (dMMR) tumors after failure of prior treatment $(11,12)$; nivolumab in combination with ipilimumab for MSI-H or dMMR metastatic colorectal cancer (mCRC) that has progressed following treatment with a fluoropyrimidine, oxaliplatin, and irinotecan (11); pembrolizumab for metastatic or advanced gastric and esophageal cancers with PD-L1 positive tumors (12). In addition to FDAapproved indications, checkpoint inhibitors have also been recommended by the National Comprehensive Cancer Network $^{\circledR}$ for second-line treatment of metastatic or advanced anal carcinoma (13).

Despite these successes, only a small percentage of patients with GI malignancies respond to immunotherapy. Effective predictive biomarkers are also lacking. Various strategies for improving response rates and survival with these agents have been evaluated in other solid tumor malignancies. For example, in patients with metastatic melanoma, the combination of checkpoint inhibitors (ipilimumab and nivolumab) was shown to improve overall survival and response rate (14). In other tumor types, the combination of chemotherapy or small molecule tyrosine kinase receptor inhibitors with checkpoint inhibitors was found to improve survival outcomes. Can some of these combination strategies be applied to GI malignancies? This article will review combination treatments employed in other tumor types to help identify current and future approaches toward improving outcomes with immunotherapy in GI malignancies.

\section{Checkpoint inhibitor combination with chemotherapy}

It is well established that traditional chemotherapy agents mediate their activity via direct cytotoxic actions. By targeting rapidly proliferating cells, cancer cells are particularly vulnerable to cell death. These agents are broadly categorized as alkylating agents (e.g., cisplatin), antimetabolites [e.g., 5-fluorouracil (5-FU), gemcitabine], topoisomerase inhibitors (e.g., irinotecan), microtubule inhibitors (e.g., paclitaxel), and cytotoxic antibiotics (e.g., doxorubicin).

In recent years, it has become increasingly clear that the effectiveness of these agents also relies upon the ability to modulate the host and tumor immune response (15). For example, 5-FU and oxaliplatin can reduce the frequency of circulating $\mathrm{T}$ regulatory (Treg) cells (16). Other agents such as gemcitabine, cisplatin, oxaliplatin and paclitaxel can increase the expression of major histocompatibility complex (MHC) class I molecules and increase the antigenicity of cancer cells $(17,18)$. Through lymphodepletion, cytotoxic chemotherapy may also force an immune system "reset" and rebound immune stimulation (19). These preclinical and clinical findings have formed the basis for combining immunotherapy and chemotherapy.

Early clinical trials with combination of chemotherapy and immunotherapy were performed in patients with small cell and non-small cell lung cancers (NSCLCs) (20,21). Both studies found an acceptable rate of adverse effects and promising clinical activity with the combination. These studies laid the foundation for use of chemotherapy in combination with immunotherapy in the first-line treatment of metastatic NSCLC and extensive-stage small cell lung cancer $(22,23)$. Similar effects were observed in patients with metastatic head and neck squamous cell cancers (HNSCC) (24). Pembrolizumab, in combination with platinum-based chemotherapy is now considered a first-line treatment option for HNSCC.

Taken together, these studies form a strong rationale for further evaluation of the combination of immunotherapy with chemotherapy in GI malignancies. Traditional chemotherapy used in GI malignancies (5-FU, oxaliplatin, cisplatin, etc.) have been shown to modulate the immune system and may be synergistic with immunotherapy. An example of a checkpoint inhibitor combination with chemotherapy in GI malignancies comes from the MODUL study. This study reported outcomes from a cohort of $445 \mathrm{mCRC}$ patients that were randomized to receive maintenance therapy with either 5-FU and bevacizumab or atezolizumab with 5 -FU and bevacizumab after induction therapy with FOLFOX (5-FU, leucovorin oxaliplatin) and bevacizumab. Unfortunately, adding atezolizumab to first-line maintenance therapy did not improve efficacy outcomes (25). Conversely, a phase II study in patients with metastatic human epidermal growth factor receptor 2 (HER2)-positive, gastric or gastroesophageal junction 
(G/GEJ) tumors found promising results. Pembrolizumab when added to first-line therapy consisting of oxaliplatin, capecitabine, and trastuzumab produced an overall response rate of $83 \%$ amongst the 24 evaluable patients (26). Grade 3-4 adverse events were uncommon. Based upon these results, a phase III study to evaluate this combination has been initiated (NCT03615326). The phase III KEYNOTE-062 trial assessed HER2-negative, PD-L1 positive, metastatic G/GEJ patients and found that the combination of pembrolizumab plus chemotherapy was not superior over pembrolizumab alone (27). Grade 3-5 drug-related adverse events were seen in $73 \%$ patients in the combination arm. Pembrolizumab was also combined with $5-\mathrm{FU}$ and cisplatin in patients with untreated metastatic gastric cancer in a cohort of the KEYNOTE-059 trial (28). The overall response rate observed was $60 \%$ in all patients compared historically to $45 \%$ with cisplatin and 5-FU alone (29). Grade 3-4 adverse events were seen in $76 \%$ of patients. A similar study combining nivolumab + chemotherapy (CheckMate 649) is currently ongoing with an anticipated completion in March 2021 (NCT02872116) (Table 1).

A phase II study of 28 patients combined pembrolizumab with chemoradiotherapy for stage I-III esophageal cancers (30). Chemoradiotherapy consisted of weekly paclitaxel and carboplatin with 44.1 Gy radiotherapy. Pathologic complete response (CR) rates were seen in $46.1 \%$ patients with most common adverse events being neutropenia (50\%) and liver enzyme elevations (31\%).

Combinations of chemotherapy and immunotherapy hold promise in GI malignancies. There are several frontline combination studies ongoing (Table 1) with eagerly anticipated results.

\section{Multiple immune checkpoint combinations}

In recent years, focus has been placed on combining inhibitors of different immune checkpoint pathways (i.e., CTLA-4 with PD-1 inhibition), especially in malignancies that have been established of having treatment responses to single-agent immunotherapy previously (31). In GI malignancies, the combination of ipilimumab and nivolumab is currently in the early stages of being evaluated in hepatocellular, esophagogastric, and colorectal cancers.

In patients with HCC previously treated with sorafenib, the combination of ipilimumab and nivolumab was evaluated in the CheckMate 040 trial. The objective response rate observed with the combination was twice of that previously seen with nivolumab monotherapy (32). In patients with previously-treated metastatic esophagogastric cancer, the combination of ipilimumab with nivolumab was evaluated in the phase I/II portion of the CheckMate 032 trial. This study included patients irrespective of PD-1/PD-L1 status and demonstrated clinical benefit, thus warranting further phase III evaluation which is currently underway (33).

Initial trials evaluating the combination of ipilimumab with nivolumab utilized a dosing regimen of ipilimumab $3 \mathrm{mg} / \mathrm{kg}$ and nivolumab $1 \mathrm{mg} / \mathrm{kg}$ every 3 weeks for 4 doses, followed by maintenance nivolumab $3 \mathrm{mg} / \mathrm{kg}$ every 2 weeks (34). A major concern with targeting multiple immune checkpoints is the added potential for immunerelated adverse events. CTLA- 4 targets T cells in the initial stages of naïve T-cell activation, whereas the PD-1 pathway targets previously activated $\mathrm{T}$ cells at later stages of the immune response (35). This earlier and expansive activity potentially accounts for the increased toxicity associated with the CTLA-4 inhibitor ipilimumab over PD-1/PDL1 inhibitors. As such, trials experimenting with doses and schedules of these agents in combination are frequently done.

In the previously treated MSI-H/dMMR mCRC patient population, an analysis of the CheckMate 142 trial using nivolumab $3 \mathrm{mg} / \mathrm{kg}$ and ipilimumab $1 \mathrm{mg} / \mathrm{kg}$ every 3 weeks for 4 doses followed by maintenance nivolumab $3 \mathrm{mg} / \mathrm{kg}$ every 2 weeks thereafter was presented. At a median 13.4 months in 119 patients, response rates of $55 \%$ were reported with $32 \%$ of patients experiencing grade 3-4 adverse effects (36). More recently, phase II data from the same CheckMate 142 trial were presented evaluating the combination of nivolumab and ipilimumab in the firstline setting. In this analysis of 45 patients, the dosing of nivolumab $3 \mathrm{mg} / \mathrm{kg}$ every 2 weeks with ipilimumab $1 \mathrm{mg} / \mathrm{kg}$ every 6 weeks continuously until progression or discontinuation was utilized. Preliminary results at a median 13.8 months indicate a comparable response rate $(60 \%)$ with only $16 \%$ of patients experiencing a grade 3-4 adverse event, potentially representing an efficacious dosing schedule that is more tolerable for patients (37).

\section{Checkpoint inhibitor combination with anti- vascular endothelial growth factor (anti-VEGF) therapy}

VEGF pathways play an integral role in angiogenesis and repair of healthy tissues (38). This pathway is found to 
Table 1 Selected ongoing studies evaluating various combination strategies with immune checkpoint inhibitors

\begin{tabular}{|c|c|c|c|c|}
\hline Disease state & Intervention & Combination & Phase & NCT number \\
\hline \multirow{2}{*}{ Anal cancer-metastatic } & Avelumab \pm cetuximab & EGFR & 2 & NCT03944252 \\
\hline & $\mathrm{mDCF} \pm$ atezolizumab & Chemo & 2 & NCT03519295 \\
\hline \multirow[t]{2}{*}{ BTC-advanced } & Durva + treme + gem or gem/cis vs. gem/cis & Chemo & 2 & NCT03473574 \\
\hline & Pembrolizumab + CapeOx & Chemo & 2 & NCT03111732 \\
\hline $\begin{array}{l}\text { Esophageal cancer- } \\
\text { metastatic }\end{array}$ & Nivolumab + ipilimumab & Dual ICl & 2 & NCT03416244 \\
\hline \multirow[t]{2}{*}{ GE cancer-resectable } & Neoadjuvant CapeOx + docetaxel + atezolizumab & Chemo & 2 & NCT03448835 \\
\hline & Perioperative atezolizumab + FLOT vs. FLOT & Chemo & 2 & NCT03421288 \\
\hline \multirow[t]{8}{*}{ GE cancer-metastatic } & Cabozantinib + durva & VEGF & 1 & NCT03539822 \\
\hline & Ramucirumab + durva & VEGF & 1 & NCT02572687 \\
\hline & FOLFOX \pm nivolumab \& ipilimumab & Chemo & 2 & NCT03647969 \\
\hline & Nivolumab + ipilimumab vs. nivolumab + chemo & Chemo/Dual ICI & 3 & NCT02872116 \\
\hline & Ramucirumab + nivolumab & VEGF & $1 / 2$ & NCT02999295 \\
\hline & Lenvatinib + pembrolizumab & VEGF & 2 & NCT03321630 \\
\hline & Pembrolizumab + oxaliplatin + capecitabine & Chemo & 2 & NCT03342937 \\
\hline & Chemotherapy \pm pembrolizumab & Chemo & 3 & NCT03675737 \\
\hline \multirow{12}{*}{ HCC-advanced } & $\begin{array}{l}\text { Durva or treme monotherapy vs. durva + treme vs. } \\
\text { Durva + bev }\end{array}$ & Dual ICI/VEGF & 2 & NCT02519348 \\
\hline & Cabozantinib + atezolizumab vs. sorafenib & VEGF & 3 & NCT03755791 \\
\hline & Atezolizumab + bev vs. sorafenib & VEGF & 3 & NCT03434379 \\
\hline & Lenvatinib + nivolumab vs. lenvatinib & VEGF & $2 / 3$ & NCT04044651 \\
\hline & Nivolumab + bev & VEGF & 1 & NCT03382886 \\
\hline & Nivolumab + ipilimumab & Dual ICl & 3 & NCT04039607 \\
\hline & Lenvatinib + nivolumab & VEGF & 2 & NCT03841201 \\
\hline & Sorafenib + nivolumab & VEGF & 2 & NCT03439891 \\
\hline & Lenvatinib + pembrolizumab vs. lenvatinib & VEGF & 3 & NCT03713593 \\
\hline & Regorafenib + pembrolizumab & VEGF & 1 & NCT03347292 \\
\hline & Sorafenib + pembrolizumab & VEGF & $1 / 2$ & NCT03211416 \\
\hline & Axitinib + avelumab & VEGF & 1 & NCT03289533 \\
\hline
\end{tabular}

Table 1 (continued) 
Table 1 (continued)

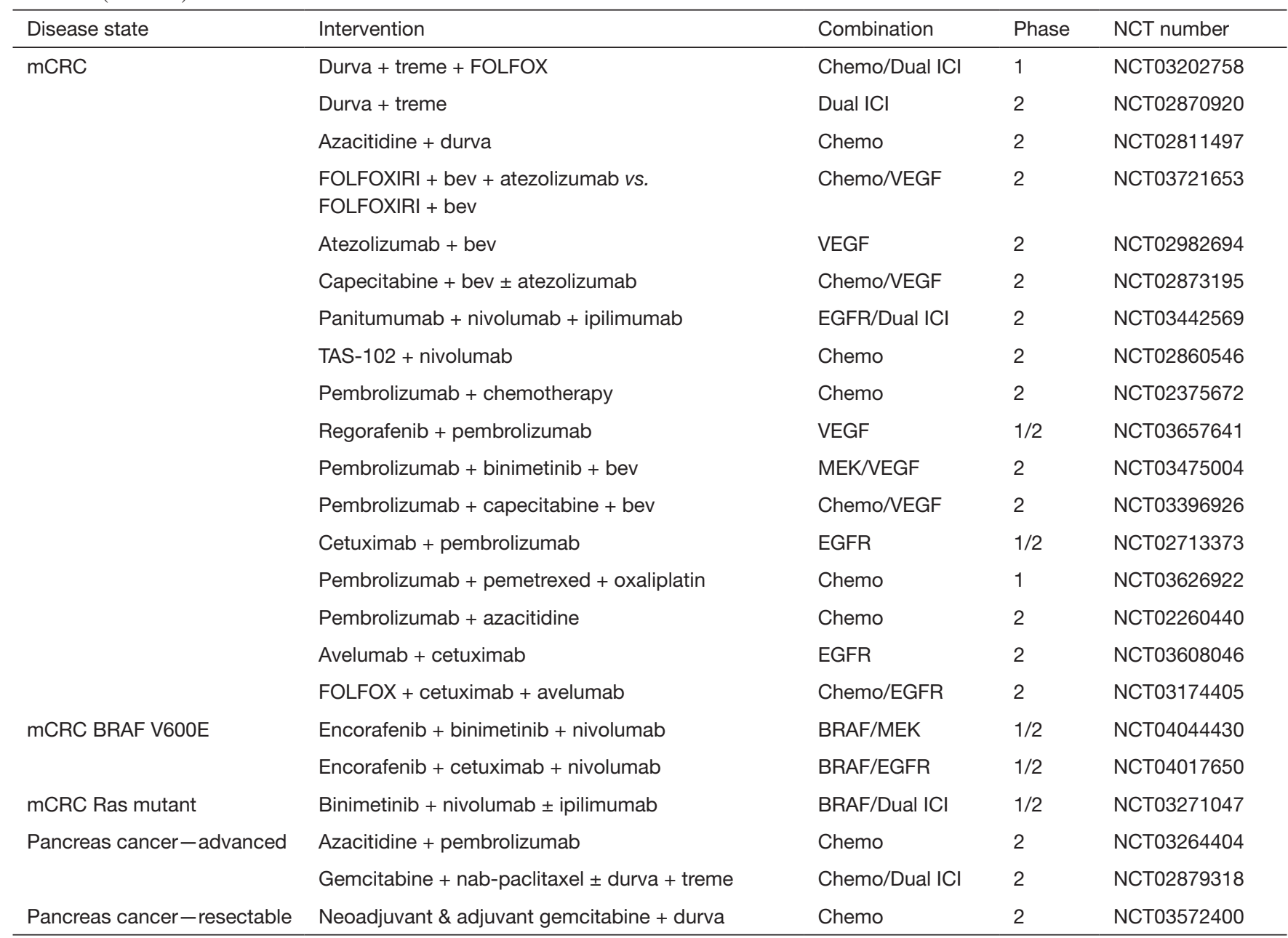

$\mathrm{ICl}$, immune checkpoint inhibitor; EGFR, epidermal growth factor receptor; mDCF, modified docetaxel + cisplatin + 5-fluorouracil; BTC, biliary tree cancer; durva, durvalumab; treme, tremelimumab; gem, gemcitabine; cis, cisplatin; GE, gastroesophageal; CapeOx, capecitabine + oxaliplatin; FLOT, 5-fluorouracil + leucovorin + oxaliplatin + docetaxel; FOLFOX, 5-fluorouracil + oxaliplatin + leucovorin; VEGF, vascular endothelial growth factor; HCC, hepatocellular carcinoma; bev, bevacizumab; mCRC, metastatic colorectal cancer; FOLFOXIRI, 5-fluorouracil + leucovorin + oxaliplatin + irinotecan.

be upregulated in numerous malignancies (39). Targeted inhibition of VEGF signaling pathways has yielded beneficial effects in various malignancies. There are currently several FDA-approved indications for antiVEGF therapies in GI malignancies. Bevacizumab, an antiVEGF antibody, has shown to improve overall survival in mCRC patients (40). Ramucirumab, a VEGF receptor (VEGFR) 2 antibody, has also been approved in second-line mCRC, gastric cancer, and esophageal cancer based upon improved overall survival demonstrated in randomized clinical trials (41). Similarly, the VEGFR-targeting tyrosine kinase inhibitors (TKI) sorafenib and lenvatinib are FDA- approved, first-line options in HCC $(42,43)$.

The VEGF pathway is also involved in regulating immune functions in the TME via several mechanisms. In vitro and animal model studies suggest that VEGF increases expression of Tregs and myeloid derived suppressor cells (MDSC) (44,45). Tregs and MDSC have shown to inhibit tumor directed T-cell responses and are generally associated with reduced inflammation and an immunosuppressive state (46). VEGFR1 also appears to interfere with dendritic cell (DC) maturation (47). Finally, animal model studies suggest a downregulation of immune stimulatory effector $\mathrm{T}$ cells in the presence of high levels of 
VEGF, similar to those observed in advanced cancer (48). The overall effect of VEGF pathway overexpression on the TME is immunosuppressive. Upregulation of VEGF compromises the endothelial vasculature and impedes the transit of T-cells to infiltrate a tumor, creating yet another barrier for immune-mediated cytotoxicity (49). Thus, combining immunotherapy with VEGF inhibitor appears to be an attractive strategy in GI malignancies.

The combination of VEGFR TKI and checkpoint inhibitors was first successfully evaluated in metastatic renal cell carcinoma. Initial studies combined checkpoint inhibitors with pazopanib or sorafenib and found grade 3-4 toxicities, especially liver function test abnormalities, to be much higher than anticipated $(50,51)$. Grade 3 elevations in alanine aminotransferase (ALT) were seen in $18 \%$ of patients treated with nivolumab plus sunitinib, $20 \%$ of patients treated with nivolumab plus pazopanib, and $60-70 \%$ of patients treated with pembrolizumab plus pazopanib. On the contrary, grade 3 ALT elevations were seen in only $8 \%$ of patients receiving axitinib and pembrolizumab (52). This could be because axitinib is a more selective inhibitor of VEGFR compared to pazopanib and sunitinib. Based upon this experience from renal cell carcinoma, it appears that combining checkpoint inhibitors with more selective VEGFR TKI therapy may be a preferred strategy from a safety standpoint. Similarly, the combination of pembrolizumab with the anti-VEGF antibody bevacizumab was found to be safe and tolerable for patients with metastatic renal cell carcinoma as well as glioblastoma $(53,54)$. In a large randomized study in patients with treatment-naive metastatic NSCLC, the PD-L1 checkpoint inhibitor atezolizumab was combined with bevacizumab and chemotherapy. This combination showed an improved response rate and a similar adverse event profile compared to the combination without atezolizumab (55). This study ultimately led to the FDA approval for the combination of atezolizumab with bevacizumab and chemotherapy as a first-line option in metastatic, non-squamous NSCLC.

A combination of durvalumab and ramucirumab was evaluated in a multi-cohort phase Ia/Ib study in solid tumors (56). Among the cohort of G/GEJ adenocarcinoma patients, an objective response rate of $36 \%(n=14)$ was noted in those with high PD-L1 positive (PD-L1 $\geq 25 \%$ of tumor cells) tumors. In contrast, an objective response rate of $0 \%(\mathrm{n}=12)$ was noted in G/GEJ patients with low PD$\mathrm{L} 1$ expression. A similar multi-cohort phase $\mathrm{Ia} / \mathrm{Ib}$ was also conducted to evaluate the combination of pembrolizumab with ramucirumab in solid tumors. Among the cohort of G/GEJ adenocarcinoma patients, an objective response rate of $7 \%(n=41)$ was noted (57). Survival was longer in the subgroup of patients with PD-L1 positive tumors $(n=22)$ compared to PD-L1 negative $(n=17)$. Both of these studies indicate that, at least in G/GEJ adenocarcinomas, encouraging response rates are seen only in PD-L1 positive tumors and a different strategy might be needed in nonPD-L1 positive tumors.

Anti-VEGF therapies are utilized across various GI malignancies and the combination of these agents with checkpoint inhibitors remains an attractive approach (Table 1).

\section{Checkpoint inhibitor combination with ErbB inhibitors}

ErbB receptor tyrosine kinases have been widely studied since the initial discovery of the epidermal growth factor receptor (EGFR) (58). The ErbB family is comprised of the HER members: EGFR/HER1/ErbB1, HER2/ErbB2, HER3/ErbB3, and HER4/ErbB4. EGFR is widely expressed in colon, rectal, and anal cancer and may be considered for patients who test negative for mutations in KRAS/NRAS/ BRAF (59). HER2, on the other hand, is overexpressed in a smaller percentage amounts of patients with gastric or esophageal cancers when compared to breast cancer. Agents including cetuximab and trastuzumab have been studied and established as efficacious in these GI malignancies.

Activation of ErbB receptors results in stimulation of downstream signaling pathways, such as MAPK, AKT, and STAT. Dimerization of ErbB receptors is known to be required for activation, which has led to investigation of drugs targeting this process (60). In addition to downstream pathway inhibition, the ErbB receptor inhibitors cetuximab and trastuzumab are known to activate immune cells, such as natural killer (NK) cells (61). It increases production of interferon gamma and stimulates antibody dependent cellular cytotoxicity. The presence of interferon gamma in the TME induces production of PD-L1, a co-inhibitory immune checkpoint. Because of the known immunemediated effects, it has been suggested that these ErbB receptor blockers in combination with $\mathrm{PD}-1 / \mathrm{PD}-\mathrm{L} 1$ blockade may have synergistic antineoplastic effects and improve response rates to treatment.

Cetuximab is currently under investigation with a variety of PD-1 and PD-L1 inhibitors in head and neck, lung, colorectal, anal, and cutaneous malignancies. Safety data has 
been presented on the combination of immunotherapy with cetuximab and chemotherapy in colorectal cancer thus far. The AVETUX trial is evaluating avelumab and cetuximab in combination with FOLFOX in previously untreated mCRC (62). At this time, the safety data imply that adverse effects did not impact the feasibility of treating with the combination. The CAVE trial is currently evaluating avelumab in combination with cetuximab in patients with mCRC who have progressed after being previously treated with chemotherapy plus cetuximab (63).

Trastuzumab is currently under investigation with a variety of PD-1 and PD-L1 inhibitors in the breast and esophagogastric cancers. A phase Ib-II study adding pembrolizumab to trastuzumab was completed in patients with trastuzumab-resistant, advanced HER2-positive breast cancer (64). The phase II portion of the study included 52 patients, $\mathrm{n}=40$ with $\mathrm{PD}-\mathrm{L} 1$-positive tumors and $\mathrm{n}=12$ with PD-L1-negative tumors. An objective response was observed in $6(15 \%)$ of the 40 patients with $\mathrm{PD}-\mathrm{L} 1$ positive tumors. No patients with PD-L1-negative tumors achieved an objective response. Adding pembrolizumab to the ErbB2 inhibitor trastuzumab demonstrated clinical benefit in patients who previously had progressed on trastuzumab therapy, warranting further expanded evaluation.

As mentioned previously in this review, the PD-1 inhibitor pembrolizumab in combination with the ErbB2 inhibitor trastuzumab and chemotherapy is initially showing efficacy in metastatic esophagogastric adenocarcinoma (26). Future studies will continue to unfold the treatment response potential of this proposed synergistic effect in GI malignancies.

\section{Checkpoint inhibitor combination with BRAF/ MEK inhibitors}

The BRAF proto-oncogene encodes for the BRAF protein which plays an important role in the EGFR-mediated MAP kinase pathway. This pathway profoundly affects cell growth, proliferation and differentiation (65). Additionally, it affects cell migration and apoptosis. Activating mutations in BRAF account for approximately $15 \%$ of mutations across various malignancies (66). The most common type of BRAF mutation is a single nucleotide mutation resulting in substitution of glutamic acid for valine (BRAF V600E) (44). Amongst GI malignancies, mutations in the BRAF gene are most commonly seen in patients with CRC (67). Approximately $10 \%$ of CRC patients carry a BRAF mutation (68). These mutations are associated with shorter progression-free survival, shorter overall survival, and overall poor outcomes. Treatment with BRAF inhibitors alone has not proven to be beneficial with a response rate of $5 \%$ (69).

BRAF mutations may give rise to an immunosuppressive state in the tumor cells. In addition, treatment with BRAF and MEK inhibitors show an increase in CD4+ and CD8+ T-cell infiltration into the TME. Based upon this rationale, pembrolizumab was combined with the MEK inhibitor trametinib and the BRAF inhibitor dabrafenib in metastatic melanoma (70). An objective response was observed in 11 patients $(73 \%)$. However, treatment demonstrated a high toxicity rate. Grade 3-4 adverse events were seen in 11 patients (73\%). Similarly, another phase I study showed high rates of hepatotoxicity $(80 \%)$ when combining ipilimumab and vemurafenib (71).

Combination therapy with atezolizumab (PD-L1 inhibitor) and cobimetinib (MEK inhibitor) was compared to regorafenib in the IMblaze370 study (50). This phase III study randomized 363 previously treated patients with mCRC to atezolizumab plus cobimetinib, atezolizumab alone, or single-agent regorafenib. After a median follow up of 7.3 months, overall survival data showed no improvement with atezolizumab or atezolizumab plus cobimetinib over regorafenib. Thus, the study failed to meet its primary endpoint. Approximately $60 \%$ of patients in the combination arm had grade 3-4 adverse effects (72). One of the criticisms of this study was the lack of biomarker driven recruitment strategy as $92 \%$ of the patients had microsatellite stable tumors (73).

These results indicate that administering BRAF/MEK inhibitors concurrently with immunotherapy may result in high rates of toxicity and novel combination strategies are needed. The disappointing results of the phase III IMblaze370 study also bring into question the one-size fits all approach with immune checkpoint combinations. Unfortunately, preclinical findings of synergy between BRAF/MEK inhibition and immunotherapy did not translate into improved clinical outcomes with this study and hence, this strategy needs revision. Some options include sequencing immunotherapy and targeted therapy and/or reducing the doses to reduce the side-effects. More biomarker-driven approaches may also be beneficial. Ongoing studies evaluating combinations of BRAF inhibitors and immunotherapy in GI malignancies are listed (Table 1).

\section{Conclusions}

Checkpoint inhibitors first made their mark in oncology 
with ipilimumab's approval in 2011 for metastatic melanoma. Since then, the applications for checkpoint inhibitors have evolved immensely and they are now approved in almost all solid tumor subtypes. More recently, novel combination strategies with a strong pre-clinical rationale have propelled forward the outcomes with immune checkpoint blockers. In GI malignancies, immune therapy has made inroads, but so far, it has only been approved as single-agent therapy in the second- or third-line setting. Approaches from other cancer types can help pave the way for future therapy in GI. Using combinations of immune checkpoint inhibitors with various other agents may potentially help unlock the resistance to immunotherapy in GI malignancies.

\section{Acknowledgments}

None

\section{Footnote}

Conflicts of Interest: Anand B. Shah declares receiving fees for serving on advisory boards from Eisai Inc., Ipsen Biopharmaceuticals, Inc. and Coherus Biosciences Inc.; Katelyn R. Sommerer has no conflicts of interest to declare; Khaldoun Almhanna declares receiving fees for serving on advisory boards from Merck.

Ethical Statement: The authors are accountable for all aspects of the work in ensuring that questions related to the accuracy or integrity of any part of the work are appropriately investigated and resolved.

\section{References}

1. Bray F, Ferlay J, Soerjomataram I, et al. Global cancer statistics 2018: GLOBOCAN estimates of incidence and mortality worldwide for 36 cancers in 185 countries. CA Cancer J Clin 2018;68:394-424.

2. Franke AJ, Skelton WP, Starr JS, et al. Immunotherapy for colorectal cancer: a review of current and novel therapeutic approaches. J Natl Cancer Inst 2019. [Epub ahead of print].

3. Saffo S, Taddei TH. Systemic management for advanced hepatocellular carcinoma: a review of the molecular pathways of carcinogenesis, current and emerging therapies, and novel treatment strategies. Dig Dis Sci 2019;64:1016-29.

4. Challis GB, Stam HJ. The spontaneous regression of cancer. A review of cases from 1900 to 1987 . Acta Oncol 1990;29:545-50.

5. Hanahan D, Weinberg RA. Hallmarks of cancer: the next generation. Cell 2011;144:646-74.

6. Topalian SL, Drake CG, Pardoll DM. Immune checkpoint blockade: a common denominator approach to cancer therapy. Cancer Cell 2015;27:450-61.

7. Bristol-Myers Squibb Company. Yervoy (ipilimumab). [package insert]. U.S. Food and Drug Administration. Available online: https://www.accessdata.fda.gov/ drugsatfda_docs/label/2018/125377s094lbl.pdf

8. Schadendorf D, Hodi FS, Robert C, et al. Pooled analysis of long-term survival data from phase II and phase III trials of ipilimumab in unresectable or metastatic melanoma. J Clin Oncol 2015;33:1889-94.

9. Rini BI, Plimack ER, Stus V, et al. Pembrolizumab plus axitinib versus sunitinib for advanced renal-cell carcinoma. N Engl J Med 2019;380:1116-27.

10. Ackermann CJ, Reck M, Paz-Ares L, et al. First-line immune checkpoint blockade for advanced non-small-cell lung cancer: travelling at the speed of light. Lung Cancer 2019;134:245-53.

11. Bristol-Myers Squibb Company. Opdivo (nivolumab). [package insert]. U.S. Food and Drug Administration. Available online: https://www.accessdata.fda.gov/ drugsatfda_docs/label/2018/125554s058lbl.pdf

12. Merck \& Co. Keytruda (pembrolizumab). [package insert]. U.S. Food and Drug Administration. Available online: https://www.accessdata.fda.gov/drugsatfda_docs/ label/2019/125514Orig1s054lbl.pdf

13. National Comprehensive Cancer Network. Anal Carcinoma (Version 1.2019). Available online: https:// www.nccn.org/professionals/physician_gls/pdf/anal.pdf

14. Wolchok JD, Chiarion-Sileni V, Gonzalez R, et al. Overall survival with combined nivolumab and ipilimumab in advanced melanoma. N Engl J Med 2017;377:1345-56.

15. Zitvogel L, Galluzzi L, Smyth MJ, et al. Mechanism of action of conventional and targeted anticancer therapies: reinstating immunosurveillance. Immunity 2013;39:74-88.

16. Maeda K, Hazama S, Tokuno K, et al. Impact of chemotherapy for colorectal cancer on regulatory T-cells and tumor immunity. Anticancer Res 2011;31:4569-74.

17. Wan S, Pestka S, Jubin RG, et al. Chemotherapeutics and radiation stimulate $\mathrm{MHC}$ class I expression through elevated interferon-beta signaling in breast cancer cells. PLoS One 2012;7:e32542.

18. Liu WM, Fowler DW, Smith P, et al. Pre-treatment with chemotherapy can enhance the antigenicity and 
immunogenicity of tumours by promoting adaptive immune responses. Br J Cancer 2010;102:115-23.

19. Bracci L, Schiavoni G, Sistigu A, et al. Immune-based mechanisms of cytotoxic chemotherapy: implications for the design of novel and rationale-based combined treatments against cancer. Cell Death Differ 2014;21:15-25.

20. Rizvi NA, Hellmann MD, Brahmer JR, et al. Nivolumab in combination with platinum-based doublet chemotherapy for fi rstline treatment of advanced non-small-cell lung cancer. J Clin Oncol 2016;34:2969-79.

21. Reck M, Bondarenko I, Luft A, et al. Ipilimumab in combination with paclitaxel and carboplatin as first-line therapy in extensive-disease-small-cell lung cancer: results from a randomized, double-blind, multicenter phase 2 trial. Ann Oncol 2013;24:75-83.

22. Langer CJ, Gadgeel SM, Borghaei H, et al. Carboplatin and pemetrexed with or without pembrolizumab for advanced, non-squamous non-small-cell lung cancer: a randomised, phase 2 cohort of the open-label KEYNOTE-021 study. Lancet Oncol 2016;17:1497-508.

23. Horn L, Mansfield AS, Szczęsna A, et al. First-line atezolizumab plus chemotherapy in extensive-stage smallcell lung cancer. N Engl J Med 2018;379:2220-9.

24. Rischin D, Harrington KJ, Greil R, et al. Protocolspecified final analysis of the phase 3 KEYNOTE-048 trial of pembrolizumab (pembro) as first-line therapy for recurrent/metastatic head and neck squamous cell carcinoma (R/M HNSCC). J Clin Oncol 2019;37:6000.

25. Grothey A, Tabernero J, Arnold D, et al. Fluoropyrimidine $(\mathrm{FP})+$ bevacizumab $(\mathrm{BEV})$ + atezolizumab vs $\mathrm{FP} / \mathrm{BEV}$ in BRAFwt metastatic colorectal cancer (mCRC): findings from cohort 2 of MODUL - a multicentre, randomized trial of biomarker-driven maintenance treatment following first-line induction therapy. Ann Oncol 2018;29:LBA19.

26. Janjigian YY, Chou JF, Simmons M, et al. First-line pembrolizumab $(\mathrm{P})$, trastuzumab $(\mathrm{T})$, capecitabine (C) and oxaliplatin (O) in HER2-positive metastatic esophagogastric adenocarcinoma (mEGA). J Clin Oncol 2019;37:62.

27. Tabernero J, Cutsem EV, Bang YJ, et al. Pembrolizumab with or without chemotherapy versus chemotherapy for advanced gastric or gastroesophageal junction (G/GEJ) adenocarcinoma: the phase III Keynote-062 study. J Clin Oncol 2019;37:LBA4007.

28. Bang YJ, Muro K, Fuchs C, et al. KEYNOTE-059 cohort 2: safety and efficacy of pembrolizumab (pembro) plus 5-fluorouracil (5-FU) and cisplatin for first-line
(1L) treatment of advanced gastric cancer. J Clin Oncol 2017;35:4012.

29. Al-Batran SE, Hartmann JT, Probst S, et al. Phase III trial in metastatic gastroesophageal adenocarcinoma with fluorouracil, leucovorin plus either oxaliplatin or cisplatin: a study of the Arbeitsgemeinschaft Internistische Onkologie. J Clin Oncol 2008;26:1435-42.

30. Hong MH, Kim HR, Park SY, et al. A phase II trial of preoperative chemoradiotherapy and pembrolizumab for locally advanced squamous cell carcinoma. J Clin Oncol 2019;37:4027.

31. Motzer RJ, Tannir NM, McDermott DF, et al. Nivolumab plus ipilimumab versus sunitinib in advanced renal-cell carcinoma. N Engl J Med 2018;378:1277-90.

32. Yau T, Kang YK, Kim TY, et al. Nivolumab (NIVO) + ipilimumab (IPI) combination therapy in patients (pts) with advanced hepatocellular carcinoma (aHCC): Results from CheckMate 040. J Clin Oncol 2019;37:4012.

33. Janjigian YY, Bendell J, Calvo E, et al. CheckMate-032 study: efficacy and safety of nivolumab and nivolumab plus ipilimumab in patients with metastatic esophagogastric cancer. J Clin Oncol 2018;36:2836-44.

34. Larkin J, Chiarion-Sileni V, Gonzalez R, et al. Combined nivolumab and ipilimumab or monotherapy in untreated melanoma. N Engl J Med 2015;373:23-34.

35. Buchbinder EI, Desai A. CTLA-4 and PD-1 pathways: similarities, differences, and implications of their inhibition. Am J Clin Oncol 2016;39:98-106.

36. Overman MJ, Lonardi S, Wong KYM, et al. Durable clinical benefit with nivolumab plus ipilimumab in DNA mismatch repair-deficient/microsatellite instability-high metastatic colorectal cancer. J Clin Oncol 2018;36:773-9.

37. Lenz HJ, Lonardi S, Zagonel V, et al. Nivolumab (NIVO) + low-dose ipilimumab (IPI) as first-line (1L) therapy in microsatellite instability-high/DNA mismatch repair deficient (MSI-H/dMMR) metastatic colorectal cancer (mCRC): clinical update. J Clin Oncol 2019;37:3521.

38. Ferrera N. Role of vascular endothelial growth factor in physiologic and pathologic angiogenesis: therapeutic implications. Semin Oncol 2002;29:10-4.

39. Goel HL, Mercurio AM. VEGF targets the tumour cell. Nat Rev Cancer 2013;13:871-82.

40. Genentech, Inc. Avastin (bevacizumab). [package insert]. U.S. Food and Drug Administration. Available online: https:/www.accessdata.fda.gov/drugsatfda_docs/ label/2017/125085s319lbl.pdf

41. Eli Lilly and Company. Cyramza (ramucirumab). [package insert]. U.S. Food and Drug Administration. Available 
online: https://www.accessdata.fda.gov/drugsatfda_docs/ label/2015/125477s011lbl.pdf

42. Bayer Inc. Nexavar (sorafenib). [package insert]. U.S. Food and Drug Administration. Available online: https://www.accessdata.fda.gov/drugsatfda_docs/ label/2018/021923s020lbl.pdf

43. Eisai Inc. Lenvima (lenvatinib). [package insert]. U.S. Food and Drug Administration. Available online: https://www.accessdata.fda.gov/drugsatfda_docs/ label/2018/206947s007lbl.pdf

44. Gabrilovich D, Ishida T, Oyama T, et al. Vascular endothelial growth factor inhibits the development of dendritic cells and dramatically affects the differentiation of multiple hematopoietic lineages in vivo. Blood 1998;92:4150-66.

45. Huang Y, Chen X, Dikov MM, et al. Distinct roles of VEGFR-1 and VEGFR-2 in the aberrant hematopoiesis associated with elevated levels of VEGF. Blood 2007;110:624-31.

46. Pere H, Tanchot C, Bayry J, et al. Comprehensive analysis of current approaches to inhibit regulatory $\mathrm{T}$ cells in cancer. Oncoimmunology 2012;1:326-33.

47. Dikov MM, Ohm JE, Ray N, et al. Differential roles of vascular endothelial growth factor receptors 1 and 2 in dendritic cell differentiation. J Immunol 2005;174:215-22.

48. Ohm JE, Gabrilovich DI, Sempowski GD, et al. VEGF inhibits T-cell development and may contribute to tumorinduced immune suppression. Blood 2003;101:4878-86.

49. Peske JD, Woods AB, Engelhard VH. Control of CD8 T-cell infiltration into tumors by vasculature and microenvironment. Adv Cancer Res 2015;128:263-307.

50. Amin A, Plimack ER, Infante JR, et al. Nivolumab (antiPD-1; BMS-936558, ONO-4538) in combination with sunitinib or pazopanib in patients (pts) with metastatic renal cell carcinoma (mRCC). J Clin Oncol 2014;32:5010.

51. Chowdhury S, McDermott DF, Henner Voss M, et al. A phase I/II study to assess the safety and efficacy of pazopanib (PAZ) and pembrolizumab (PEM) in patients (pts) with advanced renal cell carcinoma (aRCC). J Clin Oncol 2017;35:4506.

52. Atkins MB, Plimack ER, Puzanov I, et al. Axitinib in combination with pembrolizumab in patients with advanced renal cell cancer: a non-randomised, open-label, dose-finding, and dose-expansion phase $1 \mathrm{~b}$ trial. Lancet Oncol 2018;19:405-15.

53. Dudek AZ, Liu L, Shivaram Alva A, et al. Phase ib and phase II studies of pembrolizumab (P) with bevacizumab (B) for the treatment of metastatic renal cell carcinoma
(RCC): BTCRC-GU14-003. J Clin Oncol. 2018;36:4558.

54. Reardon D, Nayak L, Peters K, et al. Phase II study of pembrolizumab or pembrolizumab plus bevacizumab for recurrent glioblastoma (rGBM) patients. J Clin Oncol 2018;36:2006.

55. Socinski MA, Jotte RM, Cappuzzo F, et al. Atezolizumab for first-line treatment of metastatic nonsquamous NSCLC. N Engl J Med 2018;378:2288-301.

56. Bang Y, Golan T, Lin C, et al. Ramucirumab (Ram) and durvalumab (Durva) treatment of metastatic non-small cell lung cancer (NSCLC), gastric/gastroesophageal junction (G/GEJ) adenocarcinoma, and hepatocellular carcinoma (HCC) following progression on systemic treatment(s). J Clin Oncol 2019;37:2528.

57. Herbst RS, Arkenau HT, Santana-Davila R, et al. Ramucirumab plus pembrolizumab in patients with previously treated advanced non-small-cell lung cancer, gastro-oesophageal cancer, or urothelial carcinomas (JVDF): a multicohort, non-randomised, open-label, phase 1a/b trial. Lancet Oncol 2019;20:1109-23.

58. Chan DLH, Segelov E, Wong RS, et al. Epidermal growth factor receptor (EGFR) inhibitors for metastatic colorectal cancer. Cochrane Database Syst Rev 2017;6:CD007047.

59. Dienstmann R, Vermeulen L, Guinney J, et al. Consensus molecular subtypes and the evolution of precision medicine in colorectal cancer. Nat Rev Cancer 2017;17:79-92.

60. Ferris RL, Lenz HJ, Trotta AM, et al. Rationale for combination of therapeutic antibodies targeting tumor cells and immune checkpoint receptors: harnessing innate and adaptive immunity through IgG1 isotype immune effector stimulation. Cancer Treat Rev 2018;63:48-60.

61. Luedke E, Jaime-Ramirez AC, Bhave N, et al. Cetuximab therapy in head and neck cancer: immune modulation with interleukin-12 and other natural killer cell-activating cytokines. Surgery 2012;152:431-40.

62. Stein A, Binder M, Al-Batran SE, et al. Avelumab and cetuximab in combination with FOLFOX in patients with previously untreated metastatic colorectal cancer (MCRC): Results of the safety run-in phase of the phase II AVETUX trial (AIO-KRK-0216). J Clin Oncol 2018;36:3561.

63. Troiani T, Martinelli E, Ciardiello D, et al. Phase II study of avelumab in combination with cetuximab in pre-treated RAS wild-type metastatic colorectal cancer patients: CAVE (cetuximab-avelumab) Colon. J Clin Oncol 2019;37:TPS731.

64. Loi S, Giobbie-Hurder A, Gombos A, et al. Pembrolizumab plus trastuzumab in trastuzumab-resistant, advanced, HER2-positive breast cancer (PANACEA): a 
single-arm, multicentre, phase $1 \mathrm{~b}-2$ trial. Lancet Oncol 2019;20:371-82.

65. Jovanovic B, Kröckel D, Linden D, et al. Lack of cytoplasmic ERK activation is an independent adverse prognostic factor in primary cutaneous melanoma. J Invest Dermatol 2008;128:2696-704.

66. Davies H, Bignell GR, Cox C, et al. Mutation of the BRAF gene in human cancer. Nature 2002;417:949-54.

67. Barras D. BRAF mutation in colorectal cancer: an update. Biomark Cancer 2015;7:9-12.

68. Cancer Genome Atlas Network. Comprehensive molecular characterization of human colon and rectal cancer. Nature 2012;487:330-7.

69. Kopetz S, Desai J, Chan E, et al. Phase II pilot study of vemurafenib in patients with metastatic BRAF-mutated colorectal cancer. J Clin Oncol 2015;33:4032-8.

doi: $10.21037 / \operatorname{tgh} .2019 .09 .04$

Cite this article as: Shah $\mathrm{AB}$, Sommerer KR, Almhanna K. Immune checkpoint inhibitors in gastrointestinal malignancies: what can we learn from experience with other tumors? Transl Gastroenterol Hepatol 2019;4:73.
70. Ribas A, Lawrence D, Atkinson V, et al. Combined BRAF and MEK inhibition with PD-1 blockade immunotherapy in BRAF-mutant melanoma. Nat Med 2019 Jun;25:936-40.

71. Ribas A, Hodi FS, Callahan M, et al. Hepatotoxicity with the combination of vemurafenib and ipilimumab. N Engl J Med 2013;368:1365-6.

72. Eng C, Kim TW, Bendell J, et al. Atezolizumab with or without cobimetinib versus regorafenib in previously treated metastatic colorectal cancer (IMblaze370): a multicentre, open-label, phase 3, randomised, controlled trial. Lancet Oncol 2019;20:849-61.

73. Sclafani F. MEK and PD-L1 inhibition in colorectal cancer: a burning blaze turning into a flash in the pan. Lancet Oncol 2019;20:752-3. 